\title{
Association between daily physical activity and neighborhood environments
}

\author{
Kanae Kondo · Jung Su Lee $\cdot$ Kiyoshi Kawakubo $\cdot$ Yusuke Kataoka $\cdot$ \\ Yasushi Asami · Katsumi Mori · Masahiro Umezaki · Taro Yamauchi · \\ Hirofumi Takagi $\cdot$ Hiroshi Sunagawa $\cdot$ Akira Akabayashi
}

Received: 11 August 2008/ Accepted: 15 February 2009/Published online: 19 March 2009

(C) The Japanese Society for Hygiene 2009

\begin{abstract}
Objective Previous studies on the association between physical activity (PA) and neighborhood environments (NE) focused on either objectively measuring the NE or the residents' perception of NE. Here, we investigate which actual or perceived NE is associated with residents' PA in Japan. Methods Two regions with an objectively assessed high and low residential density, land use mix-diversity, and street connectivity, respectively, were identified in one city. The subjects were selected using a stratified random sampling method by sex and age in each region. The NE of the subjects was objectively measured using the Geographic Information System (GIS), and the subjects' perception of the NE was
\end{abstract}

K. Kondo and J.S. Lee contributed equally to this work. K. Kondo, J.S. Lee, and K. Kawakubo designed the study, collected the data, and wrote the manuscript. K. Mori analyzed the data. Y. Kataoka and Y. Asami analyzed the GIS data. M. Umezaki, T. Yamauchi,

H. Takagi, and H. Sunagawa collected the data. A. Akabayashi supervised the research.

\section{K. Kondo · J. S. Lee $(\bowtie) \cdot$ K. Mori}

Department of Health Promotion Science,

Graduate School of Medicine, The University of Tokyo,

7-3-1 Hongo, Bunkyo-ku, Tokyo 113-0033, Japan

e-mail: jslee@m.u-tokyo.ac.jp

\section{K. Kawakubo}

Department of Food Sciences and Nutrition,

Kyoritsu Women's University, Tokyo, Japan

Y. Kataoka · Y. Asami

Center for Spatial Information Sciences,

The University of Tokyo, Tokyo, Japan

\section{Umezaki}

Department of Human Ecology,

Graduate School of Medicine,

The University of Tokyo, Tokyo, Japan assessed using a questionnaire. The daily total number of walking steps was measured with an accelerometer, and walking and cycling time were assessed by a questionnaire. Results For the female subjects, the mean cycling time, subjectively assessed as a means of transport, was significantly longer in the group with a high GIS score for the number of land use types, while the score for total number of walking steps was significantly higher among those who were aware of places to walk to, and cycling time for transport was longer for those who perceived an accessibility to post offices, banks/credit unions, gymnasiums/fitness facilities, and amusement facilities in their neighborhood. For the male subjects, the score for walking time for leisure was longer for those who perceived aesthetics and an accessibility to parks, and the score for total walking steps was significantly higher for those who perceived an accessibility to bookstores or rental video stores in their neighborhood.

\author{
T. Yamauchi \\ Department of Human Ecology, \\ Graduate School of Medicine, \\ Hokkaido University, Sapporo, Japan \\ H. Takagi \\ School of Nursing, Faculty of Medicine, \\ Toho University, Tokyo, Japan \\ H. Sunagawa \\ Hagi Center for Health and Welfare, Hagi, Japan \\ A. Akabayashi \\ Department of Biomedical Ethics, \\ Graduate School of Medicine, \\ The University of Tokyo, Tokyo, Japan
}


Conclusions The results to this study demonstrate that daily PA was high among female subjects living in a $\mathrm{NE}$ with land use mix-diversity, and who had an awareness of places to walk to and the accessibility to facilities for daily necessities in their neighborhood. For male subjects, daily PA was high among those who perceived the aesthetics of and accessibility to facilities for pleasure in their neighborhood. Further research is needed to determine the association between PA and $\mathrm{NE}$ on the basis of sex differences.

Keywords Geographical Information System (GIS) . Neighborhood environment · Perception of neighborhood environment $\cdot$ Physical activity $\cdot$ Sex differences

\section{Introduction}

Previous studies have shown that low physical activity (PA) in daily life increases the incidence of cardiovascular disease, diabetes, cancer, osteoporosis, and depression [1]. Low PA has also been shown to increase mortality [2-4].

In Japan, health objectives for the year 2010, called Healthy Japan 21, were established in 2000 by the Ministry of Health, Labor and Welfare. One of the PA objectives set for adults in Healthy Japan 21 was an increase in the number of daily walking steps. However, despite this objective, the number of daily walking steps has decreased between 1997 and 2004 [5, 6]. Consequently, improvements in daily PA is an important public health issue in Japan.

Several demographical, biological, psychological, and behavioral factors have been identified to explain individual differences in PA [7]. It has also been determined that the social and physical environment also play important roles in health behavior [7]. There is a general agreement that effective public health approaches aimed at promoting PA must include modifiable environmental factors that support behavioral change [8]. However, there is a need to gain a better understanding of the environmental attributes that may influence the PA of the general population [7]. As such, the correlation between PA and neighborhood environment (NE) has recently attracted much attention [9].

In previous studies, the NE has been assessed using objective measurements or residents' perception. In this studies, objectively measured NE parameters, such as high residential density, high land use mix-diversity, high street connectivity [10-12], and accessibility to facilities [13], were positively associated with $\mathrm{PA}$ regardless of sex. However, sex differences were also reported for the association between NE and PA. Residents' perception of NE, such as aesthetics for male residents, accessibility to facilities for female residents [14], and accessibility to sidewalks for both male and female residents [15], were positively associated with PA. To date, most of the earlier studies on the association between PA and NE have focused on either objectively measured NE parameters or residents' perception of the NE. It is therefore necessary to investigate which actual or perceived NE is associated with residents' PA in Japan.

Residents' daily PA has also been assessed using objective or self-reported measurements, such as an accelerometer, which has been used in earlier studies for measuring total daily PA [16]. However, an accelerometer cannot link each event of PA with the purpose of that activity. This is particularly true for PA for leisure or transport as it is related with NE $[13,14]$. Therefore, PA needs to be assessed both objectively and separately according to the activity's purpose.

The aim of this study was to clarify the association between daily PA and NE in Japan using both objective measurements and residents' perception of NE. This is the first study of this type in Japan. Physical activity was also measured using an accelerometer and a questionnaire to distinguish the activity's purpose. Our first hypothesis was that daily PA would differ between types of regions (NE) with different objective physical characteristics; our second hypothesis was that the association between PA and NE would differ depending on the measurement methods of NE and sex of the study population.

\section{Methods}

\section{Study areas}

The study area was Hagi City in Yamaguchi Prefecture. According to the 2005 Census of Japan, the city has a relatively small population of 57,990 in a $699-\mathrm{km}^{2}$ area [17]. The percentage of residents aged 65 or older was $31.6 \%$ at the time of the census.

In previous studies, PA was found to be high among residents living in neighborhoods with high residential density, high land use mix-diversity, and high street connectivity [10, 11]. These studies had a comparative design in which two different types of regions in a community were compared; the studies did not compare different communities [18], because, if largely different communities were to be compared, the socio-cultural background of the residents could affect the results.

Therefore, in our study, we selected two types of regions according to the selection method of the previous studies. Type A was considered a region with an objectively assessed high residential density, high land use mixdiversity, and high street connectivity; Type B was 


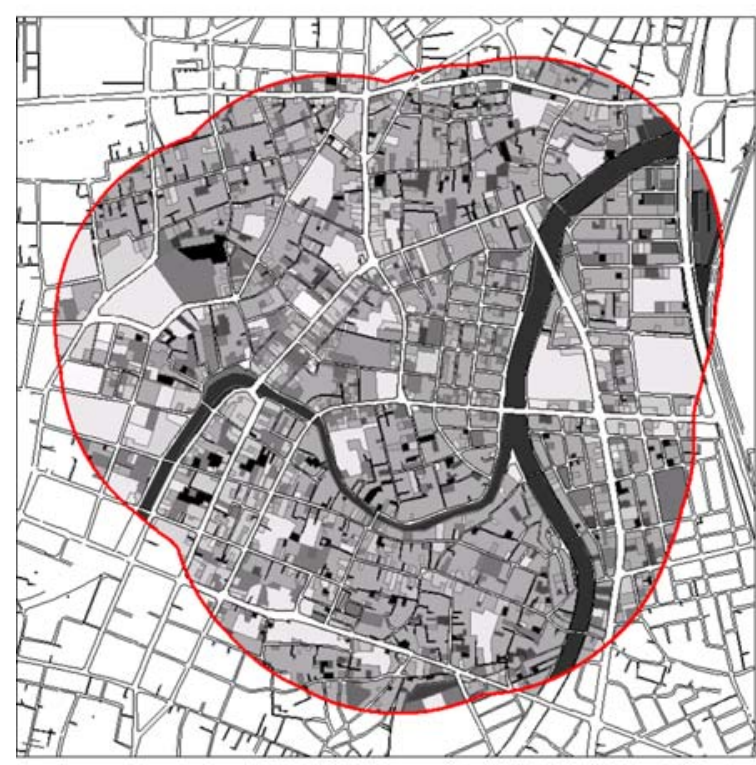

Type A region

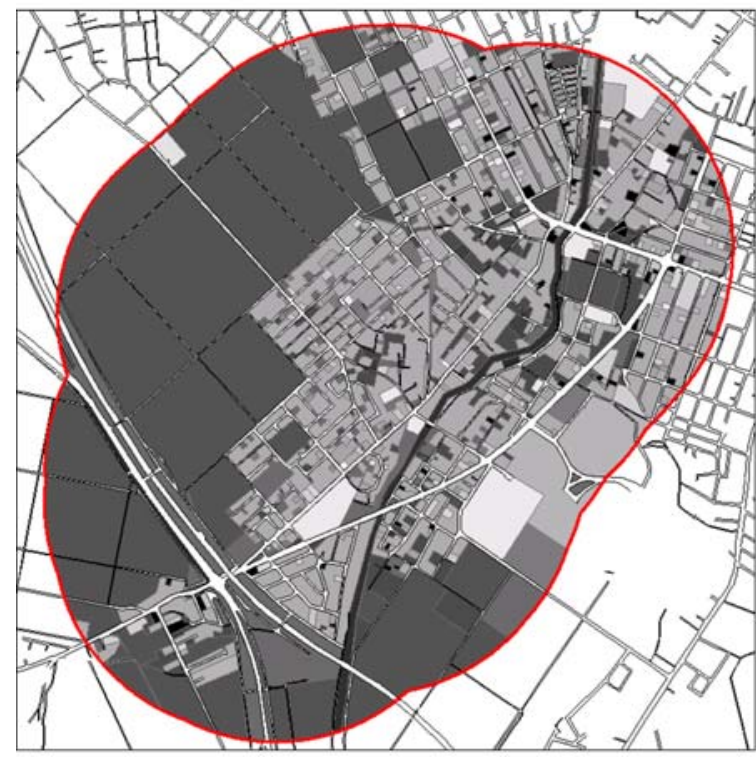

Type B region

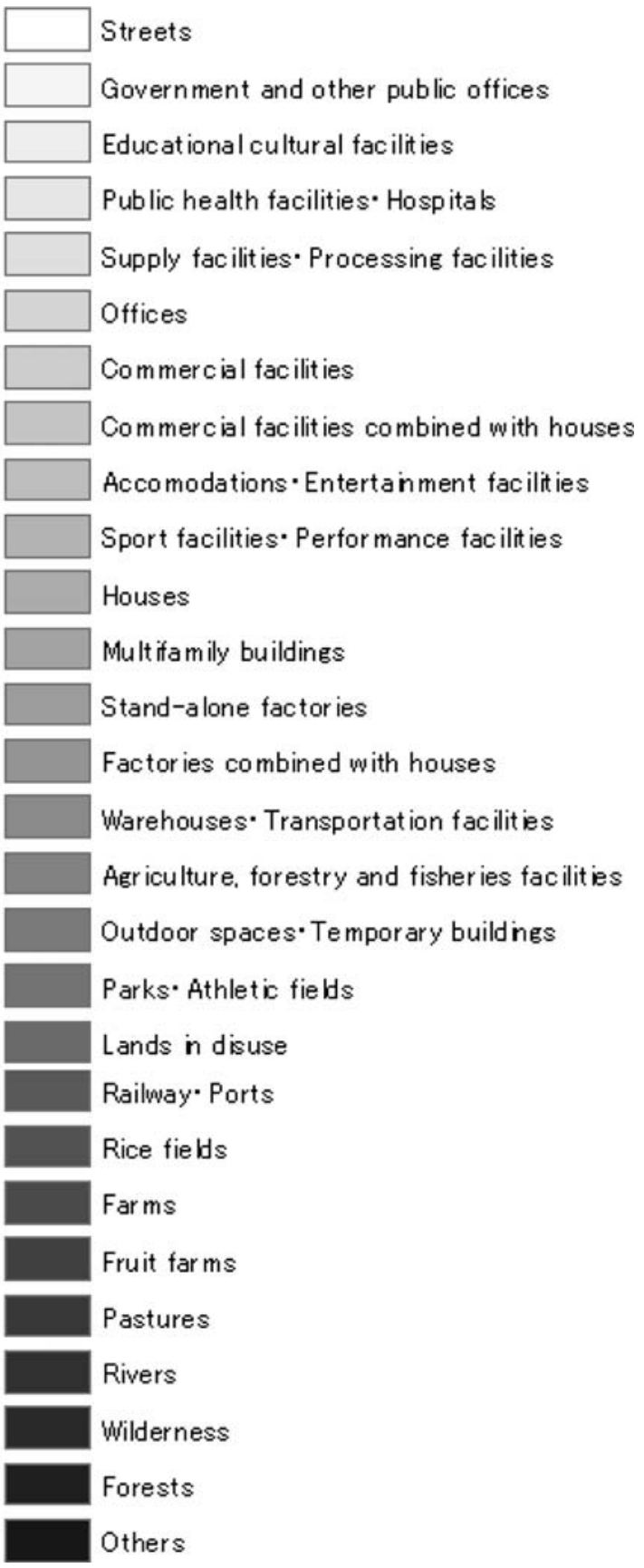

Fig. 1 Land use of the Type A and B regions

considered a region with an objectively assessed low residential density, low land use mix-diversity, and low street connectivity (Fig. 1).

Study subjects

The subjects of the study were selected using a stratified random sampling method among the residents (age range 30-69 years) of each region by sex and 5-year age strata on the basis of data from the Basic Resident Register, in
September 2006. A total of 480 subjects (240 from the Type A region and 240 from the Type B region) were selected for this study in Hagi City.

A cover letter, a written consent form, and a selfadministered questionnaire were sent to the subjects by mail. Introduction to the study, encouragement for participation, and a request to monitor daily walking using an accelerometer were included in the cover letter. The questionnaire contained items about perceived NE, PA, and demographic variables. When the signed consent form and 
the questionnaire were returned, an accelerometer was sent to the subjects in which their height, weight, and age had been inputted. The subjects were asked to wear the accelerometer for 1 week and send it back by mail.

This study was approved by the Ethical Committee of the Graduate School of Medicine, The University of Tokyo, Japan.

\section{Neighborhood environments}

\section{Objective measurements using the Geographical Information System (GIS)}

The Geographic Information System (GIS) is a combination of computer applications and databases that can be used to synthetically process and manage data on spatial locations and to express various objectively measured design characteristics of a community. In this study, GIS was used as the means to objectively measure the subjects' NE environments.

Each subject's neighborhood was defined as the area with a 500-m radius of their house. It was assumed to be a 10-min walking distance [19].

Using Arc GIS 9 (ESRI-Japan Co., Tokyo, Japan), we calculated the following NE indices for each subject's neighborhood: (1) number of households, as a quantification measure of residential density [20]; (2) number of land use types, as a quantification measure of land use mixdiversity [21]; (3) length of streets (in meters), which quantifies street connectivity [22]; (4) number of intersections, which quantifies street connectivity [22]; (5) length of sidewalks (meters); (6) width of streets (meters) [21].

There was no GIS database for the study city, and it was difficult to precisely identify types of land use with a house map; consequently, fieldwork was carried out in the study regions. Land use types were determined by two researchers in each region and classified according to the 28 types defined by the Tokyo City Planning Basic Survey [23].

\section{Residents' perception using a questionnaire}

Using a self-administered questionnaire-the Japanese translation of the Abbreviated version of the Neighborhood Environment Walkability Scale (ANEWS), which was developed for international comparisons [24]—subjects answered questions regarding their perception of their NE. ANEWS assesses several environmental characteristics: (1) residential density; (2) "land use mix-diversity", which is an indication of proximity to nonresidential land uses, such as restaurants and retail stores; (3) "land use mix-access", which indicates ease of access to nonresidential land uses, such as restaurants and retail stores; (4) "street connectivity", which indicates easy access to streets; (5) "walking places", such as sidewalks and pedestrian trails; (6) aesthetics; (7) traffic and crime safety. Each scale assessment included 6, 25, 7, 3, 4, 4, and 9 questions, respectively. Each subject's neighborhood was defined as a 10- to 15-min walking distance from their house.

On the basis of the subjects' answers for these questions, the following NE scores were calculated for each subject's neighborhood: (1) residential density; (2) land use mixdiversity; (3) land use mix-access; (4) street connectivity; (5) walking places; (6) aesthetics and (7) safety. The higher the scores, the more positive the perception of their NE was.

In order to examine the reliability and internal consistency of our results, we carried out a study using the Japanese translation of ANEWS among 72 residents of the Koto Ward in Tokyo. Pearson's correlation coefficients of the scores, which were calculated to examine the reliability of the study scores, were $0.61-0.95$, with the exception of the score for street connectivity (0.46) and the score for places for walking (0.42). Cronbach's $\alpha$ coefficients were calculated to examine internal consistency and found to be $0.57-0.94$. Therefore, reliability and internal consistency of the Japanese translation of ANEWS was moderate to high.

\section{Physical activity}

\section{Measurements using an accelerometer}

Subjects' total number of walking steps (steps/day) was measured with an accelerometer for 1 week (Suzuken Co., Aichi, Japan) [25, 26].

Data were excluded for the days when the accelerometer was worn for $<8 \mathrm{~h}$ [27] or when the exercise volume was $<50 \mathrm{kcal} /$ day. Periods of $\geq 30$ continuous min when subjects did not wear the accelerometer were defined as periods when the count was equal to zero [27]. The minimum number of datasets needed for analysis was 3 days [28].

\section{Measurements according to the purpose of the PA using a questionnaire}

The International Physical Activity Questionnaire (IPAQ) [29-31] was used to examine measures of various kinds of PA. The IPAQ was developed to allow for cross-national comparison and to provide common instruments for assessing health-related PA. Subjects answered questions about their daily PA time (min/day) in terms of leisure and transport, and time devoted to walking for leisure, walking for transport, and cycling for transport were calculated for the study. 
Statistical analysis

The Student's $t$ test was used to compare the objective measurements and residents' perception of NE between the two types of regions.

The Student's $t$ test or chi-square test was used to compare the subjects' demographic variables between the two types of regions. An analysis of covariance (ANCOVA) adjusting for age and sex was used to compare total walking steps, time of walking for leisure, time of walking for transport, and time of cycling for transport between the two types of regions.

Based on the GIS measurements or the perception scores of the ANEWS, subjects were classified as being in the high scoring group (measurement or score was equal to and above the median) or low scoring group (measurement or score was below the median). The ANCOVA adjusting for age was used to compare, according to sex, total number of walking steps, walking time for leisure, walking time for transport, and cycling time for transport between the high scoring group and low scoring group.

The subjects were further classified into the two groups according to accessibility to particular facilities within 10 min of walking distance. Facilities used for classification were post offices, bookstores, banks/credit unions, rental video stores, bus stops/stations, parks, gymnasiums/ fitness facilities, and amusement facilities. An ANCOVA adjusting for age was used to compare total walking steps, walking time for leisure, walking time for transport, and cycling time for transport between female and male subjects with and without accessibility, respectively.

Statistical analysis was conducted using the SAS statistical package, Windows version (release 9.1; SAS Institute, Cary, N.C.). A $P$ value $<0.05$ was considered statistically significant.

\section{Results}

Respondents to the study

Eighty-three subjects (35\%) from the Type A region and 73 subjects $(30 \%)$ from the Type B region answered the questionnaire; of these, 48 subjects from the former region and 64 from the latter were asked to wear an accelerometer for 1 week. Those subjects who answered the questionnaire were significantly older than those who did not (data not shown), However, there were no significant differences in the percentage of females to males. Similarly, those who wore an accelerometer were significantly older than those who did not; in addition, a higher percentage of the former were habitual exercisers. However, there were no significant differences in the mean walking time for leisure, walking time for transport, and cycling time for transport, as assessed by the questionnaire.

\section{Characteristics of NE}

Table 1 shows a comparison of the subjects' NE in the two regions. The objective GIS measurements for the number of households, length of streets, and number of intersections were significantly higher and the measurements for the length of sidewalks and width of streets were significantly lower in the Type A region than in the Type B region.

The perception score for land use mix-diversity, land use mix-access, and street connectivity were significantly higher in the Type A region than in the Type B region. The perception score for walking places was significantly lower in the Type A region than in the Type B region. There were no significant differences in the perception scores for residential density, aesthetics, and safety between the two regions.

\section{Characteristics of study subjects}

Table 1 shows the comparison of study subjects' characteristics between the Type A and Type B regions. There were no significant differences in the mean age, body mass index, percentage of female residents, and percentage of habitual exercisers between the Type A and Type B regions. Further, walking time for leisure, walking time for transport, cycling time for transport, and the mean total walking steps were not significantly different between the Type A and Type B regions.

\section{Association between PA and objective} measurements of NE

Subjects were classified into the high scoring group or the low scoring group according to the NE measurements obtained using the GIS. Table 2 compares the mean total walking steps measured with an accelerometer, and walking time for leisure and cycling time for transport using the IPAQ between the high and low scoring groups, by sex, after adjustment for age.

For both sexes, there were no significant differences in walking steps between the high scoring group and the low scoring group for the number of households, number of land use types, length of streets, number of intersections, length of sidewalks, and width of streets. In addition, there were no differences in walking time for leisure between the high scoring group and the low scoring group for all objective NE measurements for both male and female subjects.

However, for female subjects only, the mean cycling time for transport was significantly longer in the high 
Table 1 Comparison of subjects' neighborhood environments, characteristics, and physical activities between the Type A region and the Type B region

\begin{tabular}{|c|c|c|}
\hline Characteristics of neighborhoods/subjects & Type $\mathrm{A}^{\mathrm{a}}(n=73)$ & Type $\mathrm{B}^{\mathrm{a}}(n=83)$ \\
\hline \multicolumn{3}{|c|}{ Objective assessment of neighborhood environments using the GIS } \\
\hline Number of households $(n)$ & $1,039.7 \pm 132.8$ & $580.9 \pm 236.1$ \\
\hline Number of land use types $(n)$ & $22.2 \pm 0.8$ & $21.9 \pm 1.9$ \\
\hline Length of streets $(\mathrm{m})$ & $11,374.0 \pm 1,073.0$ & $6,416.6 \pm 1,896.7 *$ \\
\hline Number of intersections $(n)$ & $54.3 \pm 9.1$ & $20.0 \pm 10.1^{*}$ \\
\hline Length of sidewalks (m) & $2,207.6 \pm 1,155.6$ & $2,573.4 \pm 1,025.5^{*}$ \\
\hline Width of streets (m) & $9.5 \pm 1.8$ & $12.8 \pm 2.0^{*}$ \\
\hline \multicolumn{3}{|c|}{ Perception of neighborhood environments using the ANEWS } \\
\hline Residential density & $225.1 \pm 78.7$ & $210.0 \pm 43.2$ \\
\hline Land use mix-diversity & $3.7 \pm 0.6$ & $2.9 \pm 0.8^{*}$ \\
\hline Land use mix-access & $3.4 \pm 0.5$ & $3.0 \pm 0.6^{*}$ \\
\hline Street connectivity & $3.1 \pm 0.8$ & $2.6 \pm 0.7 *$ \\
\hline Walking places & $2.2 \pm 0.8$ & $2.5 \pm 0.6^{*}$ \\
\hline Aesthetics & $2.3 \pm 0.7$ & $2.5 \pm 0.7$ \\
\hline Safety & $2.2 \pm 0.3$ & $2.1 \pm 0.4$ \\
\hline \multicolumn{3}{|l|}{ Subjects' characteristics } \\
\hline Age (years) & $53.8 \pm 11.3$ & $51.0 \pm 11.2$ \\
\hline Height $(\mathrm{cm})$ & $161.3 \pm 8.9$ & $161.4 \pm 8.3$ \\
\hline Weight $(\mathrm{kg})$ & $58.5 \pm 10.9$ & $59.4 \pm 9.9$ \\
\hline BMI $\left(\mathrm{kg} / \mathrm{m}^{2}\right)$ & $22.4 \pm 2.9$ & $22.7 \pm 2.7$ \\
\hline Female $(\%)$ & 52.1 & 57.8 \\
\hline Ownership of cars (\%) & 90.4 & 97.6 \\
\hline Habitual exercisers $(\%)^{\mathrm{b}}$ & 37.0 & 42.2 \\
\hline Habitual smokers $(\%)^{\mathrm{c}}$ & 21.9 & 13.3 \\
\hline Habitual drinkers $(\%)^{\mathrm{d}}$ & 53.4 & 44.6 \\
\hline \multicolumn{3}{|l|}{ Physical activities using the IPAQ } \\
\hline Walking for leisure (min/day) & $4.6 \pm 3.0$ & $7.1 \pm 2.9$ \\
\hline Walking for transport (min/day) & $3.3 \pm 2.1$ & $8.0 \pm 2.0$ \\
\hline Cycling for transport (min/day) & $7.9 \pm 2.2$ & $4.5 \pm 2.1$ \\
\hline \multicolumn{3}{|c|}{ Physical activities using an accelerometer $(n=48)(n=64)$} \\
\hline Total walking steps (steps/day) & $9,364.0 \pm 567.0$ & $8,293.5 \pm 490.7$ \\
\hline
\end{tabular}

Data are given as the mean \pm standard deviation (SD), percentage, or adjusted mean \pm SE (only for PA times and walking steps). Physical activity (PA) times and walking steps were analyzed by ANCOVA adjusting for age and sex

GIS Geographic Information System, IPAQ International Physical Activity Questionnaire, ANEWS Abbreviated version of the Neighborhood Environment Walkability Scale, BMI Body mass index

$* P<0.05$; Student's $t$ test

a Type A, A region with an objective assessment of high residential density, land use mix-diversity, and street connectivity; Type B, a region with an objective assessment of low residential density, land use mix-diversity, and street connectivity

${ }^{\mathrm{b}}$ Habitual exercisers, subjects who reported exercising regularly

${ }^{c}$ Habitual smokers, subjects who reported smoking regularly

${ }^{\mathrm{d}}$ Habitual drinkers, subjects who reported drinking regularly

scoring group than in the low scoring group for the number of land use types. In comparing the mean walking time for transport (data not shown), there was no difference between the high and low scoring groups for all objective NE measurements for both sexes.
Association between PA and perception of NE

Subjects were classified into the high scoring group or the low scoring group according to each NE score using the ANEWS. 
Table 2 Comparison of daily physical activities between the high and the low scoring group according to the objective GIS neighborhood environment measurements and by sex

\begin{tabular}{|c|c|c|c|c|c|c|c|c|}
\hline \multirow[t]{3}{*}{ Characteristics of neighborhoods/physical activities } & \multicolumn{4}{|c|}{ Male subjects } & \multicolumn{4}{|c|}{ Female subjects } \\
\hline & \multicolumn{2}{|c|}{ High scoring group ${ }^{a}$} & \multicolumn{2}{|c|}{ Low scoring group ${ }^{\mathrm{b}}$} & \multicolumn{2}{|c|}{ High scoring group ${ }^{a}$} & \multicolumn{2}{|c|}{ Low scoring group ${ }^{\mathrm{b}}$} \\
\hline & $n$ & Measurement & $n$ & Measurement & $n$ & Measurement & $n$ & Measurement \\
\hline \multicolumn{9}{|l|}{ Total walking steps using an accelerometer (steps/day) } \\
\hline Number of households $(n)$ & 26 & $8895 \pm 964$ & 27 & $8594 \pm 946$ & 28 & $8722 \pm 558$ & 31 & $8798 \pm 530$ \\
\hline Number of land use types $(n)$ & 24 & $9166 \pm 776$ & 29 & $7561 \pm 1296$ & 28 & $8595 \pm 466$ & 31 & $9113 \pm 678$ \\
\hline Length of streets (m) & 24 & $9519 \pm 988$ & 29 & $8098 \pm 899$ & 29 & $9094 \pm 558$ & 30 & $8462 \pm 530$ \\
\hline Number of intersections $(n)$ & 39 & $9519 \pm 988$ & 14 & $8098 \pm 899$ & 40 & $9062 \pm 549$ & 19 & $8472 \pm 539$ \\
\hline Length of sidewalks (m) & 31 & $9566 \pm 882$ & 22 & $7580 \pm 1057$ & 31 & $8863 \pm 530$ & 28 & $8649 \pm 557$ \\
\hline Width of streets (m) & 31 & $8600 \pm 880$ & 22 & $8941 \pm 1046$ & 31 & $8233 \pm 525$ & 28 & $9348 \pm 553$ \\
\hline \multicolumn{9}{|l|}{ Walking time for leisure using the IPAQ (min/day) } \\
\hline Number of households $(n)$ & 35 & $9.9 \pm 6.3$ & 32 & $13.6 \pm 6.6$ & 41 & $1.8 \pm 1.1$ & 42 & $0.9 \pm 1.1$ \\
\hline Number of land use types $(n)$ & 34 & $12.3 \pm 5.5$ & 33 & $10.1 \pm 8.4$ & 42 & $1.3 \pm 0.9$ & 41 & $1.4 \pm 1.4$ \\
\hline Length of streets (m) & 34 & $8.3 \pm 6.4$ & 33 & $15.0 \pm 6.5$ & 42 & $1.6 \pm 1.1$ & 41 & $1.0 \pm 1.1$ \\
\hline Number of intersections $(n)$ & 47 & $8.3 \pm 6.4$ & 20 & $15.0 \pm 6.5$ & 56 & $1.7 \pm 1.1$ & 27 & $1.0 \pm 1.1$ \\
\hline Length of sidewalks (m) & 35 & $9.4 \pm 6.4$ & 32 & $14.1 \pm 6.7$ & 41 & $1.7 \pm 1.1$ & 42 & $1.0 \pm 1.1$ \\
\hline Width of streets $(\mathrm{m})$ & 34 & $12.7 \pm 6.5$ & 33 & $10.6 \pm 6.6$ & 41 & $1.0 \pm 1.1$ & 42 & $1.6 \pm 1.1$ \\
\hline \multicolumn{9}{|l|}{ Cycling time for transport using the IPAQ ( $\mathrm{min} /$ day) } \\
\hline Number of households $(n)$ & 35 & $4.2 \pm 1.7$ & 31 & $2.4 \pm 1.8$ & 41 & $6.1 \pm 2.7$ & 42 & $3.7 \pm 2.6$ \\
\hline Number of land use types $(n)$ & 46 & $3.3 \pm 1.5$ & 20 & $3.5 \pm 2.2$ & 56 & $11.9 \pm 3.0$ & 27 & $0.8 \pm 4.4^{*}$ \\
\hline Length of streets (m) & 34 & $3.9 \pm 1.7$ & 32 & $2.7 \pm 1.7$ & 42 & $9.8 \pm 3.6$ & 41 & $6.8 \pm 3.7$ \\
\hline Number of intersections $(n)$ & 34 & $3.9 \pm 1.7$ & 32 & $2.7 \pm 1.7$ & 42 & $9.9 \pm 3.6$ & 41 & $6.7 \pm 3.7$ \\
\hline Length of sidewalks (m) & 33 & $2.9 \pm 1.7$ & 33 & $3.8 \pm 1.7$ & 41 & $10.0 \pm 3.7$ & 42 & $6.7 \pm 3.6$ \\
\hline Width of streets (m) & 34 & $4.9 \pm 1.7$ & 32 & $1.7 \pm 1.8$ & 41 & $12.1 \pm 3.6$ & 42 & $4.6 \pm 3.6$ \\
\hline
\end{tabular}

Data are adjusted mean $\pm \mathrm{SE}$

$* P<0.05$; ANCOVA adjusting for age

${ }^{a}$ High scoring groups, Subjects with environment measurements using the GIS equal to and above the median

${ }^{\mathrm{b}}$ Low scoring group, Subjects with environment measurements using the GIS below the median

Table 3 shows our comparison of the mean total walking steps measured with an accelerometer, and the mean walking time for leisure and cycling time for transport using the IPAQ between the high and low scoring groups, according to sex, after adjustment for age.

For female subjects, the measurement for mean total walking steps was significantly higher in the high scoring group than in the low scoring group for the walking places score. For male subjects, the mean walking time for leisure was significantly longer in the high scoring group than in the low scoring group for the aesthetics score. There were no differences in walking steps between the high scoring group and the low scoring group for residential density, land use mix-diversity, land use mix-access, street connectivity, and safety.

There were no differences in cycling time for transport between the high scoring group and the low scoring group for all NE perception scores among both sexes.
A comparison of the mean walking time for transport (data not shown) showed that there were no differences between the high scoring group and the low scoring group for all NE perception scores among both sexes.

Association between PA and accessibility to particular facilities

Subjects were classified according to whether particular facilities were within a 10-min walking distance (Table 4). The scores for total walking steps, walking time for leisure, and cycling time for transport were compared among the subjects by sex after adjustment for age.

For male subjects, the score for mean total walking steps was significantly higher for subjects with bookstores or rental video stores in the area than for subjects without these facilities. Further, the mean walking time for leisure 
Table 3 Comparison of daily physical activities between the high and the low scoring group according to each perception score of the neighborhood environment using the ANEWS and by sex

\begin{tabular}{|c|c|c|c|c|c|c|c|c|}
\hline \multirow[t]{3}{*}{ Characteristics of neighborhoods/physical activities } & \multicolumn{4}{|c|}{ Male subjects } & \multicolumn{4}{|c|}{ Female subjects } \\
\hline & \multicolumn{2}{|c|}{ High scoring group ${ }^{a}$} & \multicolumn{2}{|c|}{ Low scoring group ${ }^{\mathrm{b}}$} & \multicolumn{2}{|c|}{ High scoring group ${ }^{a}$} & \multicolumn{2}{|c|}{ Low scoring group ${ }^{b}$} \\
\hline & $n$ & Measurement & $n$ & Measurement & $n$ & Measurement & $n$ & Measurement \\
\hline \multicolumn{9}{|l|}{ Total walking steps using an accelerometer (steps/day) } \\
\hline Residential density & 25 & $8249 \pm 993$ & 28 & $9182 \pm 937$ & 37 & $9032 \pm 484$ & 22 & $8307 \pm 629$ \\
\hline Land use mix-diversity & 28 & $9449 \pm 924$ & 25 & $7949 \pm 979$ & 29 & $9304 \pm 539$ & 30 & $8238 \pm 530$ \\
\hline Land use mix-access & 29 & $9450 \pm 897$ & 24 & $7889 \pm 986$ & 36 & $8612 \pm 491$ & 23 & $8996 \pm 615$ \\
\hline Street connectivity & 22 & $9937 \pm 1035$ & 31 & $7894 \pm 868$ & 34 & $8515 \pm 505$ & 25 & $9097 \pm 589$ \\
\hline Walking places & 36 & $9117 \pm 811$ & 17 & $7946 \pm 1180$ & 31 & $9488 \pm 511$ & 28 & $7957 \pm 538^{*}$ \\
\hline Aesthetics & 34 & $9286 \pm 831$ & 19 & $7768 \pm 1113$ & 35 & $9161 \pm 495$ & 24 & $8180 \pm 600$ \\
\hline Safety & 35 & $9315 \pm 819$ & 18 & $7627 \pm 1146$ & 29 & $9057 \pm 546$ & 30 & $8477 \pm 537$ \\
\hline \multicolumn{9}{|l|}{ Walking time for leisure using the IPAQ (min/day) } \\
\hline Residential density & 34 & $12.7 \pm 6.4$ & 33 & $10.5 \pm 6.5$ & 48 & $1.9 \pm 1.0$ & 35 & $0.6 \pm 1.2$ \\
\hline Land use mix-diversity & 34 & $15.7 \pm 6.4$ & 33 & $7.5 \pm 6.5$ & 41 & $2.2 \pm 1.1$ & 42 & $0.5 \pm 1.1$ \\
\hline Land use mix-access & 35 & $5.5 \pm 6.2$ & 32 & $18.3 \pm 6.5$ & & - & & - \\
\hline Street connectivity & 28 & $14.7 \pm 7.1$ & 39 & $9.5 \pm 6.0$ & & - & & - \\
\hline Walking places & 44 & $11.5 \pm 5.7$ & 23 & $11.9 \pm 7.8$ & 44 & $1.9 \pm 1.1$ & 39 & $0.7 \pm 1.1$ \\
\hline Aesthetics & 37 & $20.6 \pm 6.0$ & 30 & $0.6 \pm 6.7^{*}$ & & - & & - \\
\hline Safety & 42 & $13.0 \pm 5.8$ & 25 & $9.4 \pm 7.5$ & 44 & $1.2 \pm 1.1$ & 39 & $1.5 \pm 1.1$ \\
\hline \multicolumn{9}{|l|}{ Cycling time for transport using the IPAQ ( $\mathrm{min} /$ day) } \\
\hline Residential density & 34 & $3.7 \pm 1.7$ & 33 & $3.0 \pm 1.7$ & 48 & $6.0 \pm 3.4$ & 35 & $11.5 \pm 3.9$ \\
\hline Land use mix-diversity & 33 & $4.2 \pm 1.7$ & 33 & $2.5 \pm 1.7$ & 41 & $10.9 \pm 3.6$ & 42 & $5.8 \pm 3.6$ \\
\hline Land use mix-access & 34 & $4.0 \pm 1.7$ & 32 & $2.7 \pm 1.7$ & 47 & $12.2 \pm 3.3$ & 36 & $3.1 \pm 3.8$ \\
\hline Street connectivity & 27 & $5.6 \pm 1.9$ & 39 & $1.8 \pm 1.6$ & 45 & $11.4 \pm 3.5$ & 38 & $4.7 \pm 3.8$ \\
\hline Walking places & 44 & $4.3 \pm 1.5$ & 22 & $1.4 \pm 2.1$ & 44 & $10.6 \pm 3.5$ & 39 & $5.7 \pm 3.7$ \\
\hline Aesthetics & 36 & $3.6 \pm 1.7$ & 30 & $3.1 \pm 1.8$ & 51 & $8.4 \pm 3.3$ & 32 & $8.2 \pm 4.2$ \\
\hline Safety & 41 & $2.5 \pm 1.5$ & 25 & $4.8 \pm 2.0$ & 44 & $9.4 \pm 3.5$ & 39 & $7.0 \pm 3.7$ \\
\hline
\end{tabular}

Data are adjusted mean \pm SE. There were no females who walked for leisure in the low scoring groups for land use mix-access, street connectivity, and aesthetics

$* P<0.05$; ANCOVA adjusting for age

${ }^{a}$ High scoring group, subjects with environment scores of the ANEWS equal to and above the median

${ }^{b}$ Low scoring group, subjects with environment scores of the ANEWS below the median

was significantly longer for subjects with parks in the area than for subjects without parks.

For female subjects, the mean cycling time for transport was significantly longer for subjects with post offices, banks/credit unions, gymnasiums/fitness facilities, and/or amusement facilities in the area than for subjects without these facilities.

\section{Discussion}

Based on the results of this study, we have taken a first step towards clarifying the association between daily PA and NE. This is the first study in Japan where subjects' NE was simultaneously assessed by both objective measurements
(GIS) and by the subjects' perception (questionnaire). Daily PA was also assessed using an accelerometer and a questionnaire based on the objective of the specific activity. As the results of the study reveal, the perception scores for walking places and aesthetics were positively associated with the residents' daily PA.

The first hypothesis was that daily PA would differ between types of regions with different objectively assessed NE characteristics. However, there were no significant differences in daily PA measured in the study between the Type A region and the Type B region. Based on the GIS data obtained in our study, Type A and Type B regions were significantly different in terms of number of households, length of streets, number of intersections, length of sidewalks, and width of streets, except for the number of 
Table 4 Association between daily physical activities and accessibility to particular facilities within 10 min of walking distance according to $\operatorname{sex}$

\begin{tabular}{|c|c|c|c|c|c|c|c|c|}
\hline \multirow[t]{3}{*}{ Physical activities/accessibility to particular facilities } & \multicolumn{4}{|c|}{ Males } & \multicolumn{4}{|c|}{ Females } \\
\hline & \multicolumn{2}{|c|}{ Inaccessible group $^{a}$} & \multicolumn{2}{|c|}{ Accessible group ${ }^{b}$} & \multicolumn{2}{|c|}{ Inaccessible group ${ }^{a}$} & \multicolumn{2}{|c|}{ Accessible group ${ }^{\mathrm{b}}$} \\
\hline & $n$ & Measurement & $n$ & Measurement & $n$ & Measurement & $n$ & Measurement \\
\hline \multicolumn{9}{|l|}{ Total walking steps using an accelerometer (steps/day) } \\
\hline Post offices & 37 & $6848 \pm 1214$ & 16 & $9561 \pm 787$ & 42 & $8869 \pm 719$ & 17 & $8718 \pm 456$ \\
\hline Book stores & 26 & $6983 \pm 881$ & 27 & $10568 \pm 898^{* *}$ & 23 & $8509 \pm 488$ & 36 & $9156 \pm 611$ \\
\hline Banks/credit unions & 30 & $8683 \pm 1025$ & 23 & $8786 \pm 896$ & 25 & $9071 \pm 502$ & 34 & $8341 \pm 586$ \\
\hline Rental video stores & 24 & $7422 \pm 873$ & 29 & $10336 \pm 962 *$ & 22 & $8612 \pm 484$ & 37 & $9014 \pm 627$ \\
\hline Bus or train stops & 41 & $8455 \pm 1415$ & 12 & $8826 \pm 764$ & 51 & $8379 \pm 1042$ & 8 & $8822 \pm 412$ \\
\hline Parks & 28 & $7971 \pm 983$ & 25 & $9429 \pm 927$ & 27 & $9034 \pm 532$ & 32 & $8439 \pm 582$ \\
\hline Gyms or fitness facilities & 6 & $8529 \pm 708$ & 47 & $10403 \pm 1983$ & 6 & $8826 \pm 404$ & 53 & $8193 \pm 1202$ \\
\hline Amusement facilities & 20 & $8839 \pm 855$ & 33 & $8580 \pm 1102$ & 18 & $8459 \pm 455$ & 41 & $9452 \pm 687$ \\
\hline \multicolumn{9}{|l|}{ Walking time for leisure using the IPAQ ( $\mathrm{min} /$ day) } \\
\hline Post offices & 43 & $21.5 \pm 8.2$ & 24 & $11.9 \pm 6.0$ & 53 & $2.3 \pm 3.1$ & 30 & $6.3 \pm 2.3$ \\
\hline Book stores & 33 & $21.4 \pm 6.9$ & 34 & $9.5 \pm 6.7$ & 34 & $5.3 \pm 2.4$ & 49 & $4.1 \pm 2.9$ \\
\hline Banks/credit unions & 35 & $15.6 \pm 7.2$ & 32 & $15.1 \pm 6.7$ & 35 & $2.1 \pm 2.4$ & 48 & $8.7 \pm 2.8$ \\
\hline Rental video stores & 28 & $19.9 \pm 6.4$ & 39 & $9.3 \pm 7.4$ & 32 & $5.7 \pm 2.4$ & 51 & $3.4 \pm 3.0$ \\
\hline Bus or train stops & 52 & $7.8 \pm 10.8$ & 15 & $17.2 \pm 5.4$ & 67 & $1.3 \pm 4.2$ & 16 & $5.7 \pm 2.1$ \\
\hline Parks & 36 & $2.7 \pm 6.9$ & 31 & $26.2 \pm 6.4 *$ & 35 & $4.3 \pm 2.5$ & 48 & $5.7 \pm 2.9$ \\
\hline Gyms or fitness facilities & 9 & $16.2 \pm 5.2$ & 58 & $9.7 \pm 13.1$ & 8 & $5.1 \pm 2.0$ & 75 & $2.2 \pm 5.9$ \\
\hline Amusement facilities & 23 & $19.5 \pm 5.9$ & 44 & $7.1 \pm 8.4$ & 25 & $5.6 \pm 2.2$ & 58 & $3.0 \pm 3.4$ \\
\hline \multicolumn{9}{|l|}{ Cycling time for transport using the IPAQ (min/day) } \\
\hline Post offices & 42 & $3.0 \pm 3.4$ & 24 & $4.0 \pm 1.5$ & 53 & $1.5 \pm 4.2$ & 30 & $12.1 \pm 3.1 *$ \\
\hline Book stores & 32 & $6.7 \pm 2.9$ & 34 & $5.3 \pm 1.7$ & 34 & $4.8 \pm 3.3$ & 49 & $13.4 \pm 3.9$ \\
\hline Banks/credit unions & 34 & $2.8 \pm 3.0$ & 32 & $2.1 \pm 1.7$ & 35 & $3.1 \pm 3.3$ & 48 & $15.4 \pm 3.8^{*}$ \\
\hline Rental video stores & 27 & $5.9 \pm 2.7$ & 39 & $4.0 \pm 1.9$ & 32 & $4.7 \pm 3.2$ & 51 & $13.9 \pm 4.1$ \\
\hline Bus or train stops & 51 & $4.8 \pm 4.3$ & 15 & $3.3 \pm 1.4$ & 67 & $3.1 \pm 5.8$ & 16 & $9.5 \pm 2.8$ \\
\hline Parks & 35 & $4.6 \pm 3.1$ & 31 & $2.4 \pm 1.7$ & 35 & $10.1 \pm 3.4$ & 48 & $5.8 \pm 4.0$ \\
\hline Gyms or fitness facilities & 8 & $5.5 \pm 2.2$ & 58 & $0.4 \pm 3.5$ & 8 & $5.8 \pm 2.5$ & 75 & $31.9 \pm 7.8^{* *}$ \\
\hline Amusement facilities & 22 & $5.6 \pm 2.5$ & 44 & $3.3 \pm 2.1$ & 25 & $4.8 \pm 3.0$ & 58 & $16.4 \pm 4.6^{*}$ \\
\hline
\end{tabular}

Data are adjusted mean $\pm \mathrm{SE}$

$* P<0.05, * * P<0.01$; ANCOVA adjusting for age

${ }^{a}$ Inaccessible group, Subjects without particular facilities within 10 min of walking distance in their neighborhood

b Accessible group, Subjects with particular facilities within 10 min of walking distance in their neighborhood

land use types. Previous studies did report significant differences in PA time between Type A and Type B regions $[10-12,18]$. However, in all of these earlier studies, residential density, land use diversity, and street connectivity were largely different between the two comparable regions. This was also the case in our previous study in which the land use mix-diversity was largely different [12]; however, our earlier study did not have the same design as the study reported here and did not compare different regions in the same community. Thus, the differences in NE between the regions in our current study may have been too small for the measurements/scores to reveal differences in residents' PA. Further study is needed to compare residents' PA between objectively assessed different regions in Japan on the basis of the land use mix-diversity.

The second hypothesis was that the association between PA and NE would differ depending on the measurement methods of NE and sex of the study population. Gender differences were observed in the association between PA and perception scores of NE.

The total number of walking steps was significantly higher only for female subjects who were aware of places to walk to in their neighborhood relative to female subjects who had a lower awareness of walking places. A previous study reported that the awareness of neighborhood sidewalks was positively associated with walking time [15] after adjustment 
for sex. In comparing the score of perception for the walking places and the GIS measurement for the width of streets between the study regions, the perception score for walking places and the measurement for the width of streets was lower in the Type A region than in the Type B region. We conclude that in the former, there were narrow streets where pedestrians may feel too close to moving cars and may, therefore, have a low awareness of sidewalks.

For female subjects, there were no significant differences in the walking time for leisure; however, the cycling time for transport was significantly longer in the high scoring group for the objective measurement for the number of land use types and for females who perceived accessibility to post offices, banks/credit unions, gymnasiums/fitness facilities, or amusement facilities within a 10-min walk. Perception of the land use by females may differ depending on the type of land use, and they may perceive land use of facilities with daily necessary use more than those of other types. This result supports previous findings that perception of $\mathrm{NE}$ accessibility was positively associated with PA only among female subjects [14]. Females may favor walking or cycling when they perceive an accessibility to facilities for daily necessities in their neighborhood. For the male subjects, the walking time for leisure was significantly longer for those who perceived aesthetics and who perceived accessibility to parks within a 10-min walk in their neighborhood. This result supports previous findings that walking for exercise, which is a leisure activity, is positively associated with residents' perceptions of neighborhood aesthetics only for males [14]. A previous study in Japan reported that males evaluate aesthetics higher than females [32]. Therefore, males may favor their PA in places with scenic views, such as parks.

Observed sex differences of association between PA and $\mathrm{NE}$ in the current study are interesting. Walking steps and walking time for daily PA were correlated with $\mathrm{NE}$ for males, but only cycling time for daily PA was correlated with NE for females. Cycling for transport in their neighborhood is not a common behavior in Western countries. However, it is very common in Japan, specifically among females. Observed sex differences are unique correlations in Japan and are worthy of note.

There were no significant differences in all PA in this study, except for the objective NE measurement of cycling time for transport. Only cycling time for transport was positively correlated with the number of land use types for females. In a previous study, residents living in a dense area with many multi-family dwellings could easily walk to visit a neighbor; therefore, residential density may influence residents' transport [11]. However, residents in singlefamily dwellings have significantly more friends in their neighborhood than residents in multi-family dwellings in Japan [33]. In addition, a previous study reported that PA was high in residents who perceived street connectivity
[34]. An earlier study carried out in the USA selected a Type $\mathrm{A}$ region with grid streets and a Type $\mathrm{B}$ region with many cul-de-sacs. In the USA, cul-de-sacs are built according to city planning; however, there are relatively few cul-de-sacs in Japan [35] and, therefore, the results of such studies can not be applied to Japan. Furthermore, differences in the perception scores for safety between Type A and Type B regions were not significant in our study; in fact, they may have been too small to detect differences in residents' PA. Further study is needed to compare PA between residents who perceived safety in their neighborhood and residents who did not perceive safety.

The walking time for transport was not significantly different between the high scoring group and the low scoring group for any of the objective NE measurements and any NE perception. It has been reported that residents with objectively assessed accessibility to facilities walk more for transport than residents with less accessibility to facilities [13]. The percentage of car ownership (98.4\%) in our study was much higher than that reported in this previous study (74.4\%) [13]. In addition, this previous study reported that walking time for transport was significantly shorter in residents who had cars [13]. Therefore, walking time for transport was not associated with any objective measurements and any scores of perceived NE in the current study. Further study is needed to compare residents' PA between objectively and subjectively different regions in different communities in Japan.

There are several limitations to our study. First, the response rate for this study was relatively low. One possible reason for this low response rate was that participants had not only to answer the questionnaires but also to wear an accelerometer for seven consecutive days. This kind of study in other countries had a similar response rate [10]. There was a significant difference in the mean age between subjects who answered the questionnaire and those who did not, and between subjects who wore an accelerometer and those who did not. Therefore, the results of the study can be generalized for residents who are relatively old. In addition, there was a significant difference in the percentage of habitual exercisers between subjects who wore an accelerometer and those who did not. However, differences in subjects' PA measured by the IPAQ were not observed between subjects who wore an accelerometer and those who did not.

This study is cross-sectional and, therefore, it cannot confirm the causal linkage between NE and residents' PA. However, this study is the first in Japan to assess residents' NE by both objective measurements using the GIS and by perception using a questionnaire. Furthermore, daily PA was assessed using an accelerometer and a self-administered questionnaire. Therefore, the results reported here form the basis for a hypothesis for further studies in other communities of Japan. 


\section{Conclusion}

In this study, the association between daily PA and NE was clarified with both objective and subjective measurements. The study results demonstrate that daily PA was high among our female residents who live in a NE with land use mix-diversity and who have an awareness of places to walk to and accessibility to facilities for daily necessities in their neighborhood. For our male subjects, daily PA was high among residents who perceived aesthetics and had an accessibility to facilities for pleasure in their neighborhood. Further research is needed aimed at assessing PA and NE on the basis of sex differences.

Acknowledgments This study was supported by a grant from the Japan Ministry of Education, Culture, Sports, Science and Technology (ID no.17200041 and ID no. 20240063) as a part of the Study on the Evaluation of Neighborhood Environments Affecting Residents' Daily Physical Activity (Principal investigator: Jung Su LEE). The authors wish to thank the study participants.

\section{References}

1. Warburton DE, Nicol CW, Bredin SS. Health benefits of physical activity: the evidence. Can Med Assoc J. 2006;174(6):801-9.

2. Andersen LB. Relative risk of mortality in the physically inactive is underestimated because of real changes in exposure level during follow-up. Am J Epidemiol. 2004;160(2):189-95.

3. Schnohr P, Scharling H, Jensen JS. Changes in leisure-time physical activity and risk of death: an observational study of 7,000 men and women. Am J Epidemiol. 2003;158(7):639-44.

4. Kujala UM, Kaprio J, Sarna S, Koskenvuo M. Relationship of leisure-time physical activity and mortality: the Finnish twin cohort. JAMA. 1998;279(6):440-4.

5. Ministry of Health, Welfare. The national nutrition survey in Japan in 1997 (in Japanese). Tokyo: Dai-ichi Shuppan Publishing; 1999.

6. Ministry of Health, Labour, Welfare. The national nutrition survey in Japan in 2004 (in Japanese). Tokyo: Dai-ichi Shuppan Publishing; 2006.

7. Trost SG, Owen N, Bauman AE, Sallis JF, Brown W. Correlates of adults' participation in physical activity: review and update. Med Sci Sports Exerc. 2002;34(12):1996-2001.

8. Schmid TL, Pratt M, Howze E. Policy as intervention: environmental and policy approaches to the prevention of cardiovascular disease. Am J Public Health. 1995;85(9):1207-11.

9. Duncan MJ, Spence JC, Mummery WK. Perceived environments and physical activity: a meta-analysis of selected environmental characteristics. Int J Behav Nutr Phys Act. 2005;5(2):11.

10. Saelens BE, Sallis JF, Black JB, Chen D. Neighborhood-based differences in physical activity: an environment scale evaluation. Am J Public Health. 2003;93(9):1552-8.

11. Saelens BE, Sallis JF, Flank LD. Environment correlates of walking and cycling: findings from the transportation, urban design, and planning literatures. Ann Behav Med. 2003;25(2):80-91.

12. Lee JS, Kawakubo K, Kohri S, Tsujii H, Mori K, Akabayashi A. Association between residents' perception of the neighborhood's environments and walking time in objectively different regions. Environ Health Prev Med. 2006;12(1):3-10.

13. Lee C, Moudon AV. Correlates of walking for transportation or recreation purposes. J Phys Act Health. 2006;3[Suppl 1]:S77-98.
14. Humpel N, Owen N, Iverson D, Lesie E, Bauman A. Perceived environment attributes, residential location, and walking for particular purpose. Am J Prev Med. 2004;26(2):119-25.

15. Giles-Corti B, Donovan RJ. Socioeconomic status difference in recreational physical activity levels and real and perceived access to a supportive physical environment. Prev Med. 2002;35:601-11.

16. Ward DS, Evenson KR, Vaughn A, Rodgers AB, Troiano RP. Accelerometer use in physical activity: best practice and research recommendations. Med Sci Sports Exerc. 2005;37[Suppl 11]: S582-8.

17. Cabinet Office Government of Japan. Public Opinion Polls on infrastructure development (in Japanese). Tokyo: Cabinet Office, Government of Japan; 2004.

18. Frank LD, Schmid TL, Sallis JF, Chapman J, Saelens BE. Linking objectively measured physical activity with objectively measured urban form, Findings from SMARTRAQ. Am J Prev Med. 2005;28[Suppl 2]:117-25.

19. Harano M. Analysis of walking space around Kofu Station (in Japanese). Ann Hum Reg Geogr. 2007;29:51-61.

20. Statistical Bureau. Census of Japan 2(2). Tokyo: Ministry of Internal Affairs and Communication; 2001. (in Japanese).

21. Zenrin. Zenrin home map (in Japanese). Tokyo: Zenrin; 2006.

22. Japan Map Center. Digital map 25000 spatial framework (data file). Tokyo: Japan Map Center; 2006.

23. Bureau of Urban Developments Tokyo Metropolitan Government. Land use in Tokyo: 23 wards of Tokyo in the thirteen years of the Heisei period (in Japanese). Tokyo: Bureau of Citizens and Culture; 2001.

24. Cerin E, Saelens BE, Sallis JF, Frank LD. Neighborhood environment walkability scale: validity and development of a short form. Med Sci Sports Exerc. 2006;38(9):1682-91.

25. Schneider PL, Crouter SE, Lukajic O, Cassett DR. Accuracy and reliability of 10 pedometers for measuring steps over a $400-\mathrm{m}$ walk. Med Sci Sports Exerc. 2003;35(10):1779-84.

26. Crouter SE, Schneider PL, Karabulut M, Bassett DR. Validity of 10 electronic pedometers for measuring steps, distance, and energy cost. Med Sci Sports Exerc. 2003;35(8):1455-60.

27. Schmidt MD, Freedson PS, Pekow P, Roberts D, Sternfeld B, Chasan-Taber L. Validation of the Kaiser Physical Activity Survey in pregnant women. Med Sci Sports Exerc. 2006;38(1):42-50.

28. Masse LC, Fuemmeler BF, Anderson CB, Matthews CE, Trost SG, Cattellier DJ, et al. Accelerometer data reduction: a comparison of four reduction algorithms on select outcome variables. Med Sci Sports Exerc. 2005;37[Suppl 11]:S544-54.

29. Craig CL, Marshall AL, Sjostrom M, Bauman AE, Booth ML, Ainsworth BE, et al. International physical activity questionnaire: 12-country reliability and validity. Med Sci Sports Exerc. 2003; 35(8):1381-95.

30. Lee JS. Questionnaires of the assessment of daily physical activity (in Japanese). Nippon Rinsho. 2000;58[Suppl]:174-8.

31. Murase $N$, Katsumura $T$, Ueda $C$, Inoue $S$, Shimomitsu $T$. International standardization of physical activity (in Japanese). J Health Welf Stat. 2002;49(11):1-9.

32. Hasegawa K. Research on the evaluation of aesthetics and effects of regulations to maintain aesthetics (in Japanese). Tokyo: Land Researcher Development Assistance Project of the Ministry of Land, Infrastructure and Transport; 2004.

33. Kaku K. Social relationship and mutual aid (in Japanese). Kanmon Regional Research. 2007;16:33-51.

34. Atkinson JL, Sallis JF, Saelens BE, Cain KL, Black JB. The association of neighborhood design and recreational environments with physical activity. Am J Health Promot. 2005; 19(4):304-9.

35. Ieda J, Oka N. City reproduction (in Japanese). Tokyo: Gakugei Shuppannsha; 2002. 